\title{
La renovación como estrategia de desarrollo urbano en Buenos Aires (1996-2011)
}

\author{
Tomás Alejandro Guevara
}

\section{lntroducción}

Este artículo tiene como objetivo analizar las estrategias de desarrollo urbano que promovieron procesos de renovación en áreas centrales en la Ciudad Autónoma de Buenos Aires en el período 1996-2011. En él se establece que en este período, en la búsqueda de captar capitales dispuestos a invertir en la renovación de áreas centrales, se conformó un régimen urbano de desarrollo que impulsó formas de empresarialismo que tendieron a mercantilizar el espacio urbano. Esta orientación del gobierno local generó efectos socioeconómicos adversos, con importantes impactos en términos de la conservación del patrimonio arquitectónico existente. En este marco, las intervenciones públicas en el período se orientaron para generar las condiciones económicas para la renovación urbana en áreas seleccionadas para ese fin a través de un conjunto de políticas urbanas que incluyeron tanto la realización de obras de infraestructura y equipamiento como la creación de distritos especializados en distintas actividades, que incluian diversos beneficios e incentivos a la inversión privada.

Para entender lo anterior, en primer lugar, se desarrolla conceptualmente la noción de régimen urbano y se reconstruye desde una perspectiva histórica el proceso de conformación del régimen en Buenos Aires. En segundo lugar, se reconstruye la matriz de las políticas urbanas del régimen urbano de desarrollo. Posteriormente se analizan las modificaciones al Código de Planeamiento Urbano (CPU), el principal instrumento de regulación urbana de la Ciudad Autónoma de Buenos Aires (CABA), como un aspecto central del régimen urbano de desarrollo. Para concluir, se analizan las iniciativas orientadas a promover la renovación urbana en los barrios del sudeste de la ciudad (San Telmo, La Boca, Barracas y Parque Patricios).

La conformación de un régimen urbano de desarrollo en Buenos Aires

En la década de 1980 se realizaron una serie de estudios que apuntaban a desentrañar en el ámbito local las interrelaciones entre el sector público, centro del poder político y el sector privado, encarnado fundamentalmente en el capital privado, pero también, en las organizaciones de la sociedad civil. En esta línea, Stone (1989) indaga sobre el gobierno de Atlanta entre 1946-1988 y la relación entre la comunidad negra, que ocupó progresivamente las esferas de decisión política, y la comunidad de negocios, expresada en la clase alta blanca. El autor acuñó la categoría de régimen urbano para dar

1 Muchos de los argumentos de este artículo están tomados del capítulo 6 de la tesis de doctorado del autor. cuenta de las interrelaciones entre estos dos sectores y problematizar cómo las comunidades locales

* Cómo citar este artículo: Guevara, T. (2013). La renovación como estrategia de desarrollo urbano en Buenos Aires (19962011). Apuntes, 26(2), 68-79. http://dx.doi.org/10.11144/Javeriana.APC26-2.redb 


\section{La renovación como estrategia de desarrollo urbano en Buenos Aires (1996-2011)}

The renewal and urban development strategy in Buenos Aires (1996-2011)

Renovação como estratégia de desenvolvimento urbano em Buenos Aires (1996-2011)

Tomás Alejandro Guevara

tguevara@unrn.edu.ar

Doctor en Ciencias Sociales, Magíster y Especialista en Políticas Sociales y Licenciado en Sociología por la Universidad Nacional de Buenos Aires. Investigador Asistente de la Carrera de Investigador Científico del Consejo Nacional de Investigaciones Científicas y Técnicas (CONICET) con lugar de trabajo en la Sede Andina de la Universidad Nacional de Río Negro (UNRN). Profesor Adjunto de la UNRN. Miembro del Centro Interdisciplinario de Estudios sobre Territorio, Economía y Sociedad (CIETES-UNRN).

Resumen

En este artículo se analiza el despliegue de estrategias de desarrollo urbano basadas en la promoción de los procesos de renovación en áreas centrales, en el período 1996-2011, en la Ciudad Autónoma de Buenos Aires. Se establece que en este período se conformó un régimen urbano de desarrollo que impulsó formas de empresarialismo que tendieron a mercantilizar el espacio urbano, en la búsqueda de captar capitales dispuestos a invertir en la renovación de áreas centrales, sin tener en cuenta los efectos socioeconómicos adversos o los impactos en términos de la conservación del patrimonio arquitectónico. En este marco, las intervenciones públicas en el período se orientaron a generar las condiciones económicas para la renovación urbana en áreas seleccionadas para ese fin. El artículo analiza algunas de las principales intervenciones en los barrios del sudeste de la ciudad de Buenos Aires, a partir de información estadística y documentos oficiales.

Palabras clave: renovación urbana; desarrollo urbano; empresarialismo urbano; políticas urbanas patrimonio arquitectónico

Articulo de Investigación. Derivado del trabajo de Doctorado ¿La Ciudad para quién? Transformaciones

territoriales, políticas

urbanas y procesos de producción del hábitat en la Ciudad de Buenos Aires (1996-2011).

Recepción: 28 de enero de 2013

Aceptacion: 12 de abril de 2013

Disponible en línea: 20 de diciembre de 2013
Abstract

This paper analyzes the deployment of urban development strategies based on the promotion of renewal processes in central areas, in the period 1996-2011, in the Autonomous City of Buenos Aires. It is established that during this period an urban development regime was formed, it drove forms of entrepreneurship that tended to commodify urban space, seeking to raise capital willing to invest in the renewal of core areas, regardless of the adverse socioeconomic effects or impacts in terms of conservation of architectural heritage. In this context, public interventions in the period were designed to generate economic conditions for urban renewal in selected areas for this purpose. The article discusses some of the main interventions in the neighborhoods east of the city of Buenos Aires, from statistical data and official documents.

Keywords: urban renewal; urban development; urban entrepreneurialism; urban architectural heritage policies

Resumo

Neste artigo é analisado o leque de estratégias de desenvolvimento urbano baseadas na promoção dos processos de renovação em áreas centrais, no período 1996-2011, na Cidade Autónoma de Buenos Aires. Estabelece-se que neste período conformou-se um régime urbano de desenvolvimento que impulsionou formas de empreendedorismo que tenderam a mercantilizar o espaço urbano, na procura de captar capitais dispostos a investir na renovação de áreas centrais, sem levar em conta os efeitos socioeconómicos adversos ou os impactos em termos da conservação do patrimônio arquitetônico. Neste marco, as intervenções públicas no período orientaram-se a gerar as condições económicas para a renovação urbana em áreas escolhidas para tal. 0 artigo analisa algumas das principais intervenções nos bairros do sudeste da cidade de Buenos Aires, a partir de informação estadística e documentos oficiais.

Palavras-chave: renovação urbana; desenvolvimento urbano; empreendedorismo urbano; políticas urbanas, patrimônio arquitetônico

doi: 10.11144/Javeriana.APC26-2.redb 
son gobernadas. Es decir, dar cuenta de cómo en un determinado contexto global, estructural, se desarrollan las respuestas locales en los procesos de decisión implicados en el gobierno local. De ahí que sea una perspectiva adecuada para analizar la formulación e implementación de políticas urbanas y las coaliciones de gobierno locales.

El régimen urbano se refiere a una serie de arreglos, informales y formales, que se complementan para determinar la orientación del gobierno de una determinada comunidad. El régimen es instituido por una determinada coalición de gobierno que incorpora intereses diversos, incluso contradictorios, que se mantienen unidos en pos del ejercicio del poder. Por ello, las coaliciones pueden ser inestables y están en permanente reformulación. No obstante, la noción busca dar cuenta de la estabilidad relativa de estas coaliciones.

Stone (1993) identifica diferentes tipos de regímenes urbanos: 1 . De mantenimiento: quiere mantener el statu quo, su principal objetivo es proveer servicios rutinarios; 2. De desarrollo: interesado en promover el desarrollo económico; 3. Progresivo de clase media: busca protección medioambiental y control sobre las ganancias sociales que se puedan derivar del crecimiento económico; 4. De promoción de grupos desaventajados: se centra en los grupos más desaventajados política y socialmente, pero requiere de una amplia movilización de masas.

En este artículo se da a conocer la conformación de un régimen urbano de desarrollo en la ciudad de Buenos Aires, que se orientó a promover los procesos de renovación urbana en áreas centrales. Este régimen se consolidó durante la década de 1990, incluía gestiones de diferente color político y en contextos macroeconómicos cambiantes -antes y después del colapso de $2001^{2}$.

La noción de régimen urbano está emparentada con la noción de gobernanza local, proveniente de otra tradición teórico-conceptual, pero que también remite a las condiciones en que se desarrolla y hace posible el proceso de gobierno de una ciudad. Esta alude al proceso de toma de decisiones y al proceso por el que esas decisiones son implementadas o no, para lo cual se centra en los actores que están involucrados en el mismo. En ese sentido, no se limita a las instancias formales, sino que incorpora también las instancias informales de negociación y consenso de políticas. En esta línea, De Mattos (2007) señala la emergencia de la gobernanza como uno de los factores que explican la importancia creciente de los negocios inmobiliarios en el desarrollo local, lo que le permite que surja la mercantilización del desarrollo urbano. De esta manera, la gobernanza y el planeamiento estratégico reemplazan a la gestión tradicional, caracterizada por el predominio de la planificación racional, y dan forma a estilos de gestión denominadas por Harvey (2001) como empresarialismo urbano. El empresarialismo se basa, como idea central, en la alianza entre el sector público y el sector privado, donde el Estado aparece como "facilitador" de los intereses capitalistas al asumir los riesgos de la operación y así garantizar la rentabilidad del sector privado. Las intervenciones de esta alianza no están ligadas a la mejora de las condiciones de vida de la población local, sino que se relacionan mucho más estrechamente con procesos económicos no necesariamente territorializados.

En este sentido, interesa plantear que en el período de estudio se generó un tipo particular de interrelación entre el Gobierno de la Ciudad Autónoma de Buenos Aires (GCABA) y el sector privado, especialmente aquel vinculado a los desarrollos inmobiliarios, con el que se conformó un régimen urbano (o gobernanza) de desarrollo que, en aras de promover inversiones para impulsar el crecimiento económico y la creación de empleo con una lógica de empresarialismo urbano, promovió un proceso de valorización inmobiliaria en determinadas áreas de la ciudad como forma de generar las condiciones económicas para la renovación urbana.

\section{La matriz de políticas urbanas del régimen urbano de desarrollo}

La ciudad de Buenos Aires es el principal centro económico financiero del país y concentra gran parte de las inversiones inmobiliarias, lo que determina la existencia de un mercado muy dinámico. Así, desde la década de 1990 se habla recurrentemente de un "boom inmobiliario" por la cantidad de permisos de obra y metros cuadrados construidos, después de años de estancamiento. Si bien la crisis de 2001-2002 abortó temporalmente este proceso, se reinicia con gran fuerza a partir de la recuperación económica. En este marco, existe la percepción generalizada de que los precios del suelo y de los inmuebles han venido aumentando de forma acelerada, con lo
2 Desde 1998 la economía argentina ingresó en una profunda recesión que terminó con una crisis financiera en 2001, que a la vez generó una de las mayores crisis políticas de las últimas décadas. 
cual la vivienda se ha hecho cada vez más inaccesible para amplios sectores de la población. Sin embargo, no se problematiza en la misma medida las razones de este fenómeno o la incidencia de determinadas acciones $\mathrm{u}$ omisiones por parte del Estado en el mismo. Asimismo, independientemente de ser un fenómeno generalizado a toda la CABA, no parece ser homogéneo en todo su territorio, ya que existen áreas que son "seleccionadas" preferentemente para su valorización, tanto por definiciones de políticas públicas como de agentes privados. En estas áreas se promueve el desarrollo de procesos de renovación urbana, cambios de uso del suelo, refuncionalización de infraestructuras obsoletas, etc., que modifican profundamente el perfil tradicional de barrios enteros de la ciudad. Como contraparte, el Estado no instrumenta mecanismos para apropiarse de una parte del plusvalor que se genera para el desarrollo de políticas redistributivas, por lo que convalida procesos de transferencia de renta al sector privado.

En este marco, la urbanización pasa a ser liderada por capitales privados que al encarar los grandes emprendimientos inmobiliarios reemplazan el papel de "guía" que tuvo tradicionalmente el capital público, capaz de contrarrestar, con grandes inversiones, la lógica de la rentabilidad privada. Este tipo de urbanización convierte pedazos enteros de la ciudad en objeto de negocios privados, no como mero sustento de actividades, sino negocios en cuanto a la valorización del suelo y su localización en el aglomerado. La consecuencia es la transferencia de renta urbana y su apropiación por parte del sector privado -propietarios y especialmente desarrolladores urbanos- con escasos efectos en términos de desarrollo económico, social y urbano para el resto de la población. En este sentido, es una política que pone un desmesurado acento en el componente físico de lo urbano, en detrimento de otras dimensiones tanto o más importantes. Se vale para ello de estrategias de marketing urbano y de la generación de "marcas" a partir de la imagen de las ciudades, como forma de aumentar la competitividad y de captar más inversiones por parte de los capitales extranjeros.

Las diferentes intervenciones que enmarcamos en este régimen urbano de desarrollo varían en sus modalidades y formulaciones. En algunos casos se hace referencia al eufemismo de "puesta en valor" de ciertas zonas de la ciudad, sin que por ello se explicite una política pública con objetivos, programas específicos y organismos responsables de implementarla, como sucede en cualquier otra sectorialidad. En general, la expresión alude a la concentración de la inversión pública en infraestructura, equipamiento comunitario o espacio público en algún área particular, que es definida como prioritaria para su desarrollo urbano. Otras veces para ello también es necesaria la modificación de la normativa urbana vigente para viabilizar la operación. El supuesto tácito es que el desarrollo urbano así entendido ha de impulsar y promover el desarrollo económico y social del área en cuestión, lo que supone un reduccionismo arquitectónico con poco sustento.

En ese sentido, todo se desarrolla como si la "puesta en valor" fuera un objetivo deseable en sí mismo. En general, no se establecen criterios sobre qué sectores deben beneficiarse de dichas intervenciones o si es necesario que el Estado recupere algo del plusvalor generado para reinvertirlo en otros sectores o áreas. Tampoco se proponen elementos que permitan evaluar la implementación de la política, como indicadores sociales o económicos, que puedan ser medidos a lo largo del tiempo. El único indicador "objetivo" que parece permitir evaluar el impacto de dichas políticas es la evolución del precio del suelo y los inmuebles, donde el aumento los precios reflejaría el "éxito" de la "puesta en valor", sin importar quién se los apropie. Esta puesta en valor puede impactar sobre el costo de vida general por el aumento de los precios en las locaciones, servicios y bienes, y derivar en un desplazamiento poblacional de los sectores más vulnerables en términos socioeconómicos para ser reemplazado por sectores de ingresos medios-altos y altos, con lo cual se configuran procesos de gentrificación. Asimismo, de no existir una fuerte regulación estatal, el mantenimiento y conservación del patrimonio arquitectónico del área pueden verse amenazados (Troitiño, 2003).

Uno de los elementos centrales del régimen urbano de desarrollo está vinculado a la adaptación de la normativa urbana a las demandas del sector inmobiliario para promover la inversión privada en el sector. Dicha normativa es objeto recurrente de presiones y lobbies por parte de los sectores vinculados a los negocios inmobiliarios para aprobar excepciones y modificaciones. Estas relaciones entre las instancias de planificación urbana de la ciudad y el sector inmobiliario son 
constitutivas del régimen urbano de desarrollo planteado. También, ha sido recurrentemente objeto de disputa por parte de sectores vecinalistas, ambientalistas o patrimonialistas que quieren, al contrario, limitar el negocio inmobiliario en pos del cuidado del medio ambiente, la preservación de los perfiles barriales tradicionales y el patrimonio arquitectónico.

Tal vez ningún proceso ilustre mejor la preeminencia de los intereses inmobiliarios y la conformación de un régimen urbano de desarrollo que las sucesivas modificaciones al Código de Planeamiento Urbano (CPU) de la CABA. Desde su sanción, en 1977, recibió recurrentes críticas de sectores vinculados a la actividad inmobiliaria por la supuesta rigidez que había introducido en el sector. Una de las formas de paliar esta situación fue la reiterada aprobación de excepciones y el mecanismo de generación de normas especiales contempladas para parcelas superiores a los 5.000 $\mathrm{m}^{2}$ que requerían de la sanción de normativas específicas por parte del Concejo Deliberante. Más allá de las críticas a las operatorias de excepción y de normas especiales, y a la corrupción involucrada, las excepciones y las normas especiales planteaban una discusión sobre cómo adecuar el CPU a la realidad cambiante y tal vez la posibilidad de elaborar un nuevo código (Clichevsky, 1996). Así, las excepciones al cPU fueron numerosas, incluso después de su reforma, desde 1983 hasta su prohibición en 1991.

En diciembre de 1989 se sancionaron con apoyo político unánime las normas, con lo cual se flexibilizó el CPU. Esta modificación sentó las bases normativas para los procesos de renovación urbana que se sucedieron durante la década siguiente. El nuevo cPU buscaba conformar un código flexible que pudiera "incorporar las señales del mercado", en un momento en el que se trataba de reactivar la economía en su conjunto y la construcción en particular, como uno de sus principales motores. El nuevo cPU reforzó así las tendencias del mercado, el resultado fue el incremento de la construcción en las áreas de mayor concentración, con lo que aumentó la congestión de sus infraestructuras, mientras que no se tenían en cuenta otras áreas despobladas y poco densas, aunque contaran con infraestructura disponible. De esta manera, el perfil de la actividad inmobiliaria que se impulsaba poco tenía que ver con el déficit habitacional existente y con la potencial demanda habitacional, sino que estaba vinculado al rol de la actividad como promotor del crecimiento económico. Muchas de las modificaciones al CPU señaladas, apuntaban a aumentar la edificabilidad de los distritos residenciales para hacer más rentables los emprendimientos inmobiliarios.

Al poco tiempo de ser autonomizada la ciudad Buenos Aires ${ }^{3}$, y en un contexto de creciente deterioro económico y social se volvieron a hacer sentir las presiones para adaptar el cPu a la nueva situación económica, el cual volvió a ser reformado con la sanción de la Ley 449 de 2000. La flexibilidad fue, otra vez, una de las principales demandas por parte del lobby inmobiliario que se enfrentó principalmente al interés de los vecinos que querían preservar la identidad, el patrimonio arquitectónico y el medio ambiente de sus barrios tradicionales.

En esta ocasión, uno de los ejes principales de la discusión fue la necesidad de renovar la zona sur, sistemáticamente relegada por las políticas públicas y que fue calificada como área de desarrollo prioritario (Rodríguez, Bañuelo y Mera, 2008). Para ello, se habilitaron: la construcción de torres de perímetro libre y de hasta 24 metros de altura, la instalación de centros comerciales en lugares previamente destinados a galpones o depósitos, una densificación adicional del 25\% para las zonas residenciales, así como la incorporación de los convenios urbanísticos como instrumentos de flexibilización de la gestión urbana (Szajnberg y Cordara, 2007) 4 .

En términos generales, implicó un aumento de la capacidad constructiva en la zona, favoreciendo la dinámica inmobiliaria. Pero en ningún momento se especificaba para qué se habría de producir dicha recuperación, a favor de qué sectores, cómo se iba a garantizar cierta conservación del patrimonio arquitectónico en esas áreas y si el Estado iba a recuperar algo de lo que invirtiera allí a través de impuestos y gravámenes para volcarlo en políticas que compensaran los efectos negativos de la renovación de dicha área de la ciudad. Al contrario, se propusieron una serie de exenciones impositivas y subsidios para promover la inversión.

En la actualidad está pendiente el debate por la formulación de un nuevo cPU que debe adaptarse al recientemente sancionado Plan Urbano Ambiental (PUA), según establece la Constitución de la Ciudad en su artículo No. 295. En los últimos años, la verticalización, la destrucción del patrimonio arquitectónico, la transformación del
3 La municipalidad de la ciudad Buenos Aires cambió su estatuto jurídico-político por el de Ciudad Autónoma en el marco de la reforma constitucional de 1994. Como consecuencia, sancionó su propia Constitución y eligió su primer Jefe de Gobierno en 1996.

4 Estos autores enumeran las modificaciones introducidas: posibilitó "enrases" para completar el tejido al premiar con más superficie construible si se alcanza la altura de edificios linderos, determinó la figura del Área de Desarrollo Prioritario con premios de $25 \%$ sobre el FOT (Factor de Ocupación Total) en el sur, amplió al $50 \%$ la cantidad de dormitorios que en las viviendas pueden ventilarse e iluminarse a través de patios auxiliares, incorporó la figura del convenio urbanístico, aplicable a casos como la reconversión residencial de inmuebles en distritos industriales y de equipamiento con superficies mayores a $2.500 \mathrm{~m}^{2}$.

5 Más allá de alguna referencia al mismo, el PUA no tiene mayor interés como regulación de la actividad inmobiliaria y solo tiene valor como objetivación de un modelo de ciudad deseada por parte de las instancias de planificación urbana y de los sectores que participan en las mismas, porque no contempla ningún tipo de normativa o regulación concreta, sino que es meramente declamativo. 
perfil tradicionalmente residencial de baja densidad de barrios enteros, la escasez de espacios verdes y la deficiente fiscalización de las obras hacen previsible un nivel de conflicto elevado y una creciente presión en pos de una restricción mayor de la capacidad constructiva. No obstante, la oposición así planteada entre construcción indiscriminada versus patrimonio arquitectónico, espacios verdes o barrios residenciales deja fuera una discusión mucho más amplia sobre el derecho a la ciudad, la función social del suelo urbano y el acceso a la vivienda y el hábitat para todos los sectores sociales en condiciones dignas.

\section{El impulso a la renovación urbana en los barrios del sudeste: La Boca, San Telmo, Barracas y Parque Patricios}

En esta sección se reconstruye cómo se ha dado impulso a los procesos de renovación urbana en los barrios del sudeste de la ciudad -específicamente San Telmo, La Boca, Barracas y Parque Patricios (ver figura 1)- a través de iniciativas públicas puntuales, como la creación de polos y distritos industriales, la inversión en espacio público e infraestructura, la promoción de la actividad turística y cultural, exenciones tributarias, subsidios a las tasas de interés de los créditos, permisos para aumentar la capacidad constructiva, etc.

El marco general de estas intervenciones es la definición de la zona sur como área de desarrollo prioritario por parte del GCABA, lo que implicó la orientación de las políticas públicas con una "hipótesis de gestación de nuevas centralidades urbanas" (Rodríguez, Arqueros, Rodríguez et al., 2011). En este marco, el GCABA

Figura 1. Comunas y barrios de la Ciudad Autónoma de Buenos Aires Fuente: Gobierno de la Ciudad Autónoma de Buenos Aires www.buenosaires.gob.ar impulsó la creación de distritos o polos que promovieron una mayor especialización territorial de los barrios analizados. La creación de estos distritos persigue un triple objetivo: la promoción de alguna industria estratégica, el desarrollo de una zona postergada y ubicar a la ciudad de Buenos Aires en el tablero de los inversores nacionales e internacionales. No es una estrategia que se agote en los cuatro barrios seleccionados -se aplica en el Polo Farmacéutico de Lugano, otro barrio del sur-, y ni siquiera en la zona sur -el Polo Audiovisual de ColegialesPalermo-, pero sin dudas su implementación fue muy intensa en estos barrios. Esta política, publicitada con entusiasmo por la gestión, genera muchos interrogantes sobre su impacto general sobre la estructura urbana, el patrimonio arquitectónico y las condiciones de acceso al hábitat para diferentes sectores sociales. En algunos casos, el resultado no parece ser otro que aumentar la rentabilidad de empresas prexistentes que se relocalizan, y así se genera un marco propicio para el desarrollo de emprendimientos inmobiliarios privados rentables y la promoción de una discutible especialización territorial.

De no mediar políticas específicas de regulación del mercado de suelo, la concentración de la inversión pública en un territorio delimitado puede generar un proceso de valorización inmobiliaria selectiva, que se exprese en un aumento de los precios de los inmuebles, no solo en términos absolutos - cosa más bien habitual en ciclos económicos expansivos, por los denominados movimientos estructurales generales de los precios del suelo (Jaramillo, 2009)-, sino también en términos relativos, en relación con otras zonas de la ciudad y con respecto al promedio general de la misma. Los procesos de renovación urbana pueden tener efectos sociales y económicos muy negativos para la población asentada en determinada área, lo que puede determinar su desplazamiento, el desarrollo de procesos de gentrificación y la destrucción del patrimonio arquitectónico.

Las políticas analizadas no conforman una iniciativa única, sino que son un conjunto de medidas, acciones y omisiones que determinan la decisión del Estado de promover la renovación en zonas definidas como degradadas, como forma de promover el desarrollo urbano, la actividad económica y la creación de empleo. Estos procesos son todavía incipientes y no han prosperado más allá de sectores puntuales de los barrios seleccio- 
nados. Sin embargo, claramente se inscriben en la matriz de políticas públicas urbanas impulsada por el régimen urbano de desarrollo identificado. Tampoco son privativos de instancias gubernamentales tradicionalmente ligadas al planeamiento y el desarrollo urbano, como la Secretaría de Planeamiento, pues el Ministerio de Desarrollo Económico también juega un rol fundamental en la promoción de la actividad económica en esta zona, pero con un abordaje que tiene fuertes impactos territoriales.

En términos generales, la renovación urbana de los barrios del sudeste se plantea como una extensión del área central, de la misma forma que lo hizo Puerto Madero ${ }^{6}$, y que se prevé que sea asumida por Solares de Santa María ${ }^{7}$. El proyecto de renovación urbana de la zona sur comenzó por incorporar a los barrios de San Telmo y La Boca, pero también se está extendiendo paulatinamente hacia Barracas y Parque Patricios. Estos barrios, a su vez, van adquiriendo diferentes perfiles: San Telmo y La Boca están más vinculados al turismo y la gastronomía, Barracas parece más vinculado a la localización residencial de sectores de ingresos medios altos -una gentrificación en el sentido más clásico- y a la localización de un distrito administrativo, mientras que Parque Patricios se plantea como un sector especializado en establecimientos de Tecnologías de Información y Comunicación, en una estrategia de descongestión del área central (Figura 2).

La definición de la zona sur como el área de vacancia más importante de la ciudad es producto de procesos sociales y espaciales de larga data, vinculados al proceso de urbanización de la CABA. No obstante, la normativa urbana reforzó en gran medida esta tendencia al localizar allí los usos del suelo menos rentables, siguiendo la lógica de la zonificación. Este sesgo fue particularmente claro en el caso del cPU de 1977. Los primeros indicios en la prensa sobre el programa de renovación urbana aparecen en los medios gráficos hacia principios de la década de 1990 (Di Virgilio, 1999), pero pueden ser rastreados en diagnósticos y relevamientos previos, como el concurso "20 ideas para Buenos Aires" convocado en la década de 1980. En los medios gráficos se puede ver el paralelismo que se trazaba con otras experiencias de renovación, como el barrio Soho de Nueva York, y también se planteaban antimodelos que era necesario evitar, como la "guetificación" del Bronx, en esa misma ciudad.

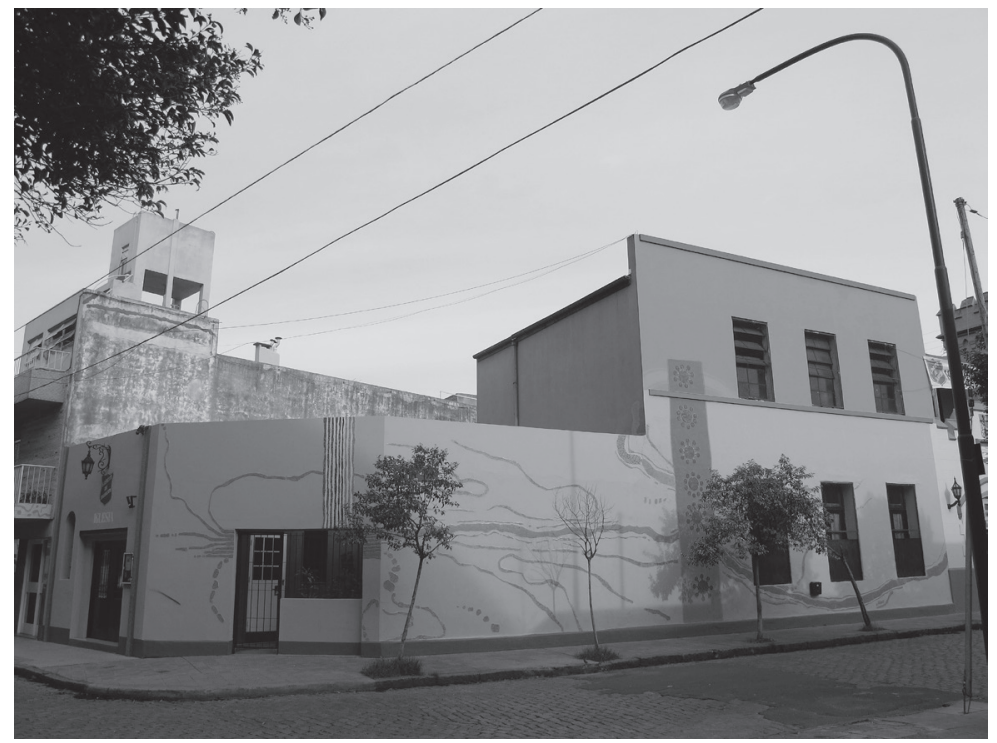

En este contexto, los barrios del sudeste han venido protagonizando transformaciones producto de la orientación territorial de las políticas públicas. Este proceso se profundizó a partir de la autonomización de la ciudad, que permitió ganar mayores grados de libertad en términos de definición de políticas de desarrollo urbano. El Plan Estratégico Buenos Aires 2010, publicado en 2005, hace especial hincapié en las diferencias territoriales entre el norte y el sur y tiene entre sus lineamientos estratégicos la “integración equitativa de la zona sur”, en donde cuestiones como el hábitat y la hidráulica del control de las inundaciones son elementos fundamentales.

Asimismo, el Plan Urbano Ambiental (PUA) ${ }^{8}$ plantea esta área como una de las más degradadas de la ciudad pero, a la vez, como la que mayores potencialidades tiene para generar un proceso de reestructuración y renovación. Según reza el documento final, en el contexto de globalización se plantea la necesidad de que Buenos Aires se inserte en el concierto de ciudades como una ciudad global y aumente su competitividad para atraer inversiones que generen desarrollo económico. Así, se impulsa la consolidación del rol internacional de Buenos Aires mediante el desarrollo de grandes equipamientos e infraestructuras, de los cuales una parte importante se alojarán en la zona sur.

Ante este diagnóstico recurrente, desde mediados de la década de 1980 el gasto público comenzó a concentrarse progresivamente en esta zona de la ciudad. Según Rodríguez, Bañuelos y Mera (2008), entre 1988 y 1997 el gasto pasó del
Figura 2.

Los Colores de Barracas

Fuente:

Pablo Vitale
6 Barrio recientemente creado producto de la renovación urbana en los viejos docks de Puerto Madero, liderada por la Corporación Antiguo Puerto Madero S.A.

7 Proyecto de desarrollo inmobiliario en el predio de la antigua Ciudad Deportiva de Boca Juniors, lindera con la Reserva Ecológica de la Costanera Sur, próxima a los barrios de Puerto Madero y La Boca.

8 La formulación del puA fue establecida por la Consartículo $29^{\circ}$, y reglamentada a través de la Ley $71 \mathrm{de}$ 1998. El órgano encargado de su formulación, el Consejo del PUA, elaboró en 2000 el Documento Final, que fue remitido a la Legislatura en 2001. Pero fue sancionado modificaciones mediante la Ley 2.930 de 2008. titución de la caBA, en su finalmente con algunas 
9 Que comprende los barrios de La Boca, Barracas, San Telmo y Montserrat.

10 Solo existen algunos datos presentados para el periodo 2006-2010, pero se refieren a una porción muy acotada del presupuesto. Las cuentas de inversión publicadas por el GCABA no tienen información específica sobre las inversiones, en el mejor de los casos, solo muestran datos de ejecución por programa.

$11 \mathrm{El}$ único de toda la serie que presentó información territorializada del gasto.

12 La línea para la zona sur abarca más barrios que los seleccionados en este trabajo, coincide con los barrios que están bajo la órbita de la Corporación Buenos Aires Sur SE, por lo que también incluye Mataderos, Villa Lugano, Villa Riachuelo, Villa Soldati, Nueva Pompeya. Además, excluye al barrio de San Telmo. El monto máximo es de hasta $\$ 600.000$, con tasa de interés fija, combinada o variable. El subsidio de tasa corre por cuenta del , y asciende a alrededor de 10 puntos porcentuales de costo financiero total.

13 Se puede observar cómo la política de valorización señalada se entrecruza y retroalimenta con políticas culturales y económicas más en general. Es una definición política que trasciende con mucho las instancias más estrechamente vinculadas al planeamiento urbano, de ahí la importancia de conceptualizarlo como un régimen urbano de desarrollo.
6\% al 15\% en el Distrito IV ${ }^{9}$; mientras que en 1999 por cada $\$ 100$ invertidos en el norte, se gastaban $\$ 130$ en el sur.

Para tener una aproximación a la distribución territorial de la inversión pública se pueden analizar los Planes Plurianuales de Inversión (PPI) disponibles en el sitio web del GCABA. Estos planes ofrecen una aproximación a la orientación territorial de la inversión pública. Sin embargo, deben ser tomados con reserva ya que no necesariamente todas las inversiones son ejecutadas y finalizadas en tiempo y forma. No obstante, pueden servir como aproximación, pues el GCABA no presenta sistemáticamente datos sobre distribución geográfica del gasto ${ }^{10}$. Si se toman los años 2003, 2005, 2007, 2009 y 2011 se puede observar que la inversión prevista en la zona sur (comunas 4 y 8) representa el 50,8\% de la inversión promedio de todo el período. Si se toma la comuna 4 de manera aislada, que comprende tres de los cuatro barrios seleccionados, el promedio para esos años representa el 34,8\%, y es, por lejos, la comuna que más inversión concentra. Este promedio esconde una disminución gradual de la inversión prevista en el sur que cae del 55,5\% entre 2003-2007, al 43,4\% para el período 2009-2011 y puede estar más vinculada a un mayor componente de iniciativas pro-mercado en el período 2009-2011 -fundamentalmente exenciones e incentivos que no requieren gastos- que a la inversión real en obras públicas.

Estos datos son coherentes con los presentados en el Programa general de acción de gobierno 2005-2007 ${ }^{11}$, en donde se indicaba que en 2005 la inversión total en la zona sur era del 51,24\% (contra tan solo el 8,25\% en la zona norte), en función de la definición de localizar la mayor parte de las inversiones en la zona sur para "producir una inyección correctiva de las disparidades que el mercado genera en la asignación de recursos". Entre las obras principales se incluyen la mencionada extensión de la Línea H (\$140 millones), la extensión de la Línea $A$-que en nuestro cálculo no se incluyó en la zona sur- (\$34 millones), la construcción de viviendas y las obras vinculadas a la reurbanización de las villas (alrededor de \$200 millones), las obras hidráulicas de la cuenca del Arroyo San Pedrito (\$23 millones) y la cuenca Boca-Barracas (\$27,5 millones), la remodelación del antiguo edificio Alpargatas para localizar la Secretaría de Justicia y Seguridad, etc. También se tomaron otras iniciativas orientadas a motorizar la demanda en el mercado inmobiliario, donde se destaca la creación de una línea de crédito hipotecario con tasa subsidiada por parte del Banco Ciudad, para la zona sur, y otra para el Distrito Tecnológico ${ }^{12}$.

A continuación, entonces, se reseñan algunas de las iniciativas impulsadas en estos barrios.

En el barrio de La Boca, el programa de Recuperación del Hábitat de La Boca (RECUP), formulado en la década de 1980, planteaba una recuperación urbana integral del barrio. Para ello se articulaban fuertes inversiones en infraestructura y espacio público con políticas de rehabilitación y consolidación del hábitat popular (Guevara, 2010). La más importante de estas inversiones, sin dudas, eran las obras de control de inundaciones de la ribera, uno de los principales déficits estructurales. Finalmente, lo que estaba pensado como una intervención integral terminó fragmentándose y se hizo hincapié en el componente hidráulico por encima del resto. La obra, que se ejecutó durante buena parte de la década de 1990 fue inaugurada en 1998, y tuvo un costo algo menor a los $\$ 80$ millones. En ese año, además, se anunció el Plan Director de Ordenamiento Hidráulico de la Ciudad, que daba el marco para realizar obras hidráulicas en diferentes cuencas, en el cual la cuenca Boca-Barracas se mantuvo como uno de sus componentes. De esta forma, la atención de la problemática de las inundaciones en La Boca fue uno de los ejes centrales de la política pública urbana en el período analizado. Todavía en el Plan de Inversiones 2003-2005 había obras de desagües por más de \$50 millones en esta cuenca.

El otro eje importante de inversión pública en el barrio fue la renovación de los espacios públicos y verdes, entre los que se destacan la Avenida Pedro de Mendoza, la creación del Parque de Flor Nativa Benito Quinquela Martín, la renovación de la Avenida Regimiento Patricios, la renovación del Paseo Garibaldi. Muchas de estas obras tenían el objetivo de extender el circuito turístico existente hacia el interior del barrio, vinculándolo con la cancha del Club Atlético Boca Juniors y su entorno. Existe en la actualidad un proyecto para la creación del Distrito de las Artes, que abarcaría buena parte del barrio, y que tendría centro en la Usina de la Música, recientemente reciclada (más de $\$ 55$ millones) ${ }^{13}$. Todas estas obras apuntaban a conectar el barrio con un área central extendida -fundamentalmente a 
través del turismo y los servicios y a partir de una recuperación pintoresca y exótica de la cultura popular que caracteriza al barrio.

Por su parte, San Telmo, desde la década de 1980, y especialmente de la de 1990, es objeto de políticas de promoción turística y cultural que tienen como objetivo recuperar su patrimonio arquitectónico. En realidad, la primera intervención en este sentido data de la década de 1970, cuando se incorporó en el cPU el Distrito U24 (Catedral al Sur - San Telmo - Avenida de Mayo) como área de preservación arquitectónica y cultural. Originalmente incluía 113 manzanas, pero fue reducida a 43 por la presión de los intereses inmobiliarios, en la medida en que se pasó de una protección arquitectónica a una protección basada en una dimensión intangible del patrimonio que busca maximizar el rédito político a partir de una mínima inversión (Rodríguez, Bañuelos y Mera, 2008). Según estos autores, a lo largo de la intervención, las políticas de preservación patrimonial transitaron desde una impronta fuertemente arquitectónica a un enfoque más intangible del patrimonio, con el fin de generar espacios atractivos para los emprendimientos inmobiliarios y turísticos ${ }^{14}$.

Estas intervenciones se enmarcan en la estrategia de posicionamiento de la CABA en el mercado turístico global. En el Programa de Acción de Gobierno 2009-2011 se incorporaba como uno de los objetivos "instalar la Marca Ciudad", con la idea de "innovar en el desarrollo de productos turísticos, lograr el posicionamiento de nuevos mercados y generar un compromiso público-privado para el desarrollo sostenible de la ciudad". Con esa finalidad se crearon el Ministerio de Turismo de la Ciudad y el Ente de Turismo que impulsaron diferentes iniciativas en este sentido, como la realización de grandes eventos culturales, sin incentivar necesariamente la producción artística preexistente en los barrios (Rodríguez, Arqueros, Rodríguez et al., 2011).

En términos de política de patrimonio arquitectónico sobresale la reciente sanción de la ley 3.686 de Recuperación de Edificios Existentes que apunta a flexibilizar las obras de renovación. Su objeto es dotar de mayor amplitud y flexibilidad a las obras de ampliación y reciclaje en edificios construidos con anterioridad al CPU de 1977. Si bien no está acotada a San Telmo, está pensada para que sus efectos principales se den en el área central y los barrios adyacentes en donde está lo- calizada la mayor parte del stock edilicio afectado por esta normativa.

En Barracas se puede mencionar una importante iniciativa, todavía incipiente, para promover el desarrollo urbano en un área bien localizada del barrio: el proyecto Centro Cívico Siglo XXI o Distrito Gubernamental. Este proyecto consiste en la relocalización de gran parte de las oficinas de la administración pública local al predio que hoy ocupan los hospitales neuropsiquiátricos Borda, Moyano y Tobar García. Este proyecto tiene dos objetivos: por un lado, generar una operación inmobiliaria con los inmuebles que hoy ocupan estas oficinas, tales como el Edificio del Plata, situado en las inmediaciones del obelisco de Buenos Aires. Por el otro, impulsar el desarrollo urbano en una zona deprimida por la presencia de las instituciones neuropsiquiátricas. Para ello es necesaria la relocalización de estas instituciones, operación que se articula con un cambio de paradigma que impulsa la tercerización y privatización de las funciones de salud mental. Recientemente se ha enviado a la Legislatura el proyecto de ley para vender el Edificio del Plata (Exp. 2222-J-2011), con la particularidad de que al mismo tiempo se prevé el alquiler de dichas oficinas al nuevo dueño por un plazo máximo de cinco años. A su vez, están en proceso de licitación los primeros edificios que albergarán a las nuevas oficinas. Solo para la construcción de la Sede Central del distrito se prevé una inversión de más de $\$ 250$ millones. El Centro Cívico Siglo XXI forma parte de un objetivo considerado estratégico por el GCABA en los diferentes Programas de Acción de Gobierno del período estudiado: la descentralización administrativa y la revalorización del espacio público.

Otra de las iniciativas importantes realizadas en el barrio es la promoción de un Distrito de Diseño, cuya sede, el Centro Metropolitano de Diseño, se construyó en el edificio remodelado del Mercado del Pescado, que implicó una inversión de más de $\$ 60$ millones.

Finalmente, en el barrio de Parque Patricios se pueden destacar dos intervenciones fundamentales que están interrelacionadas: la extensión de la Línea $\mathrm{H}$ de subterráneo y la promoción del Distrito Tecnológico.

En primer lugar, la construcción de la línea H del subterráneo, que fue aprobada en 1999 por la Ley 317 pero se inauguró en octubre de 2007, tenía como objetivo conectar esta zona de la ciudad con el área central para promover su
14 San Telmo y Montserrat llegaron a contar con más de 800 edificios catalogados dentro de este esquema de protección.

15 Recientemente el GCABA lanzó las licitaciones para las seis estaciones restantes que completan la Línea $\mathrm{H}$ desde Corrientes hasta Retiro.

16 Si bien es conocido como el Distrito Tecnológico Parque Patricios, en realidad abarca una parte considerable del barrio de Nueva Pompeya, y partes menores de los barrios de Boedo, Constitución y Barracas.

17 Incluye empresas vinculadas a la enseñanza de TIC, desarrollo, actualización, implementación, puesta en marcha, soporte y asistencia de productos de software, prestación de servicios informáticos vinculados a procesos de negocios, producción de hardware, entre otros.

18 Ver www.cai.mdebuenosaires.gob.ar 
desarrollo. Si bien el trazado completo llega hasta la zona de Retiro, actualmente solo llega hasta la avenida Corrientes donde se articula con el conjunto de la red preexistente ${ }^{15}$. La mencionada ley también estipuló que la construcción de la línea debía comenzar por el sur. En 2011, según el Plan Plurianual de Inversiones 2012-2014, el GCABA llevaba invertidos más de $\$ 580$ millones en la construcción de esta línea, y tenía previsto una inversión total de \$3.457 millones, cifra que implica por mucho la inversión más importante del período, pues representa un 12,7\% del total de inversión prevista.

Por otro lado, la creación en 2008 del Distrito Tecnológico (Ley 2.972) ${ }^{16}$ prevé una serie de incentivos y beneficios para la radicación de empresas de Tecnología de Información y Comunicación ${ }^{17}$ : exenciones al Impuesto sobre los Ingresos Brutos por el plazo de 10 años, exención total o parcial del Impuesto al Sello, exención del pago de las Contribuciones de alumbrado, barrido y limpieza, territorial y pavimentos y aceras por 10 años, exención del pago de Derechos de Delineación y Construcciones, subsidios no reintegrables para financiar hasta el 50\% del costo de la obtención de certificaciones de calidad, líneas de crédito preferenciales del Banco Ciudad, un 25\% de FOT adicional para desarrollos inmobiliarios vinculados a Tic (Ley 3.516 de 2010) (Guevara, Imori y Ronis, 2011). Según el mismo GCABA, la iniciativa está inspirada en la experiencia del “Distrito @22” de Barcelona, así como en el distrito "Caohejing Hi-Tech Parck" de Shanghai ${ }^{18}$.

Desde entonces, se buscó promover la radicación de empresas de Tecnología de Información y Comunicación en dicha zona con el objetivo de hacer de Buenos Aires la "Capital Latinoamericana de la Tecnología”. El Plan de Acción de Gobierno 2012-2014 señalaba que había más de 90 empresas radicadas en el distrito, mientras que en enero de 2012 ya había más de cien empresas radicadas. En la mayor parte de los casos no se trata de empresas recientemente creadas, sino que son empresas que se relocalizaron por los beneficios implicados.

Como parte de la promoción de este distrito se inició la construcción de la nueva sede corporativa del Banco Ciudad en una manzana ubicada entre las calles Uspallata, Atuel, Los Patos e lguazú, con un área de $35.000 \mathrm{~m}^{2}$ y un costo de \$162,4 millones más $\$ 13,6$ millones por el terreno, y la apuesta a desarrollar un proyecto de arquitectura emblemático. También se prevé la construcción del Centro Metropolitano de Tecnología, un emprendimiento público-privado que se instalará en inmuebles de la CBAS (Corporación Buenos Aires Sur). Para el período 2012-2014 se prevé una inversión de más de \$16 millones en el mejoramiento del espacio público en el polígono del Distrito Tecnológico.

El gCABA define a Parque Patricios, previamente a la creación del Distrito, como un lugar deprimido, con muchos espacios vacíos y galpones aptos para la instalación de nuevos emprendimientos (ver Distrito Tecnológico de la Ciudad de Buenos Aires. 2008-2011). Pese a esto se lo caracterizaba como con una localización privilegiada por su accesibilidad con el área central. La conformación del Distrito prevé la creación de 20 mil puestos de trabajo. Es interesante destacar que en informes oficiales se destaca que uno de los efectos relevantes de la medida fue la elevación del precio de los terrenos y oficinas, si bien se mantuvo muy por debajo de las áreas de oficinas tradicionales del centro o las más renovadas como Puerto Madero.

\section{Conclusiones}

En este artículo se reconstruyó el proceso de valorización inmobiliaria como la expresión de un régimen urbano de desarrollo conformado en la CABA a lo largo de la década de 1990. Este régimen estructuró una matriz de políticas urbanas orientadas a la promoción de la actividad inmobiliaria como palanca del crecimiento económico y de la creación de empleo a escala local. En este sentido, el régimen urbano no involucra solo a las instancias gubernamentales, sino también a una multiplicidad de actores sociales que conforman una coalición gobernante local, que lo emparentan con la noción de gobernanza urbana. En el interior de dicha matriz de políticas urbanas, la flexibilización de la normativa urbana y la desregulación de los mercados funcionan como catalizadores de las inversiones privadas.

La política de valorización inmobiliaria implica un reacomodamiento de la estructura de rentas del suelo urbano que promueve los procesos de renovación urbana y permite la apropiación privada de los diferenciales de renta generados, sin contraparte alguna para el Estado. Por su parte, los efectos en términos de desarrollo económico, social y urbano para el resto de la población 
son escasos y se pueden generar procesos sociales y económicos adversos, como el encarecimiento del costo de vida y el desplazamiento poblacional de los sectores más desfavorecidos para ser reemplazados por sectores más acomodados, lo que conforma procesos de gentrificación. A su vez, genera múltiples interrogantes en términos de su impacto en la conservación y gestión del patrimonio arquitectónico preexistente, toda vez que se plantea de forma explícita la renovación urbana de áreas completas de la ciudad. En este artículo se reconstruyeron algunas de las principales iniciativas orientadas a la promoción de la renovación urbana en los barrios del sudeste de la ciudad de Buenos Aires.

\section{Referencias}

Clichevsky, N. (1996). Politica social urbana: Normativa y configuración de la ciudad. Buenos Aires: Espacio Editorial.

De Mattos, C. (2007). Globalización, negocios inmobiliarios y transformación urbana. Nueva Sociedad, 201, 82-96.

Distrito Tecnológico de la Ciudad de Buenos Aires (2008-2011). Recuperado en www.cai. mdebuenosaires.gob.ar

Diéguez, G. y Tella, G. (2006, septiembre). Patrimonio y desarrollo inmobiliario. Ponencia presentada en VIIl Congreso Internacional de Rehabilitación del Patrimonio Arquitectónico y Edificación, Buenos Aires - Salta, Argentina.

Di Virgilio, M. (1999). El proceso de renovación del barrio de La Boca. Carta Económica Regional, 67, julio-agosto.

Guevara, T. (2010). Políticas habitacionales y procesos de producción del hábitat en la Ciudad de Buenos Aires. El caso de La Boca. Tesis de maestría en Políticas Sociales, Facultad de Ciencias Sociales, Universidad de Buenos Aires.
Guevara, T., Imori, M. y Ronis, M. (2011). Parque de los Patricios. Cosacov, N. et al. (2011, abril). Barrios al sur: Villa Lugano, Villa Riachuelo, Mataderos, Parque Patricios y Villa Soldati a través del tiempo. DT 56, 86-110, l1GG.

Harvey, D. (2001). Espacios del capital. Hacia una geografía crítica. Madrid: Akal.

Jaramillo, S. (2009). Hacia una teoría de la renta del suelo urbano. Bogotá: UniAndes.

López, E. (2008). Destrucción creativa y explotación de la brecha de la renta: discutiendo la renovación urbana del peri-centro sur poniente de Santiago de Chile entre 1990 y 2005. Scripta Nova, 12, 270. Disponible en http://www.ub.edu/geocrit/sn/sn-270/ sn-270-100.htm

Rodríguez, C., Bañuelos, C. y Mera, G. (2008). Intervención - no intervención: ciudad y políticas públicas en el proceso de renovación del Área Sur de la Ciudad de Buenos Aires. En: Herzer, H. (org.), Con el corazón mirando al sur. Transformaciones en el sur de la ciudad de Buenos Aires (pp. 45-96). Buenos Aires: Espacio.

Rodríguez, C., Arqueros, S., Rodríguez, F. et al. (2011). La política urbana "pro": continuidades y cambios en contextos de renovación en la ciudad de Buenos Aires. Cuaderno Urbano, 11, 101-121, octubre.

Stone, C. (1989). Regime Politics? [¿Políticas de Régimen?] Lawrence: Kansas Univ. Press.

Stone, C. (1993). Urban and the capacity to govern: A political economy approach. Journal of Urban Affairs, 1 (15), 1-28.

Szajnberg, D. y Cordara, C. (2007). El Código de Planeamiento Urbano de Buenos Aires o la quimera de la planificación: la densificación vertical de la Avenida Pedro Goyena, en Caballito y Flores. Café de las Ciudades, 54.

Troitiño, M. A. (2003). Renovación urbana: dinámicas y cambios funcionales. Perspectivas urbanas, 2, 1-11.

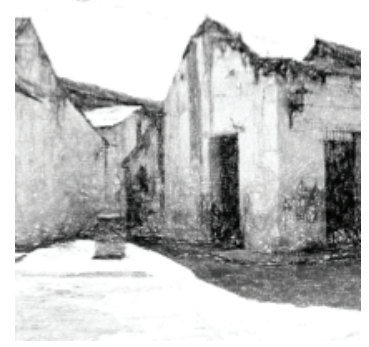

